\title{
Simposio
}

\section{Medicina Centrada en la Persona}

\section{Educación Médica Centrada en la Persona en el siglo XXI}

\author{
Juan Enrique Mezzich ${ }^{1}$
}

\section{Introducción}

El siglo XXI está emergiendo como el siglo de la persona, particularmente con respecto a la atención de salud. La perspectiva de una Medicina y Salud Centradas en la Personas se va afianzando con amplias bases históricas y ha venido madurando desde 2008 a través de dos vertientes significativas, a saber, la Medicina Clínica Centrada en la Persona y la Salud Pública Centrada en la Comunidad ${ }^{(1-4)}$.

La Medicina Centrada en la Persona (MCP) promovida por el Colegio Internacional de Medicina Centrada en la Persona (ICPCM) a través de encuentros anuales entre instituciones globales de salud, proyectos investigativos y publicaciones académicas ha ido sentando las bases conceptuales de colocar a la persona y su contexto como centro de la salud y meta de la atención de salud. La MCP propone una medicina informada por la evidencia, la experiencia y los valores y orientada a la persona en su totalidad. En esta perspectiva, la ciencia es instrumento esencial mientras que el humanismo es la esencia de la medicina. La MCP promueve una medicina de la persona (y su salud total, de la enfermedad a la calidad de vida), para la persona (promoviendo el cumplimiento del proyecto vital de cada quien), por la persona (cultivando al profesional de la salud como persona cabal, con compromiso ético y científico), y con la persona (colaborando respetuosamente con la persona que se presenta en busca de ayuda) ${ }^{(5)}$.

La Salud Pública Centrada en la Comunidad ha sido promovida principalmente por la Organización Mundial de la Salud (OMS). La publicación del Reporte sobre la Salud Mundial 2008 titulado Atención primaria de salud: ahora más que nunca ${ }^{(2)}$ menciona prominentemente en su introducción que "Los valores de atención primaria de salud para lograr salud para todos requieren sistemas de salud que pongan a las personas en el centro de la atención médica", recogiendo de esta manera la propuestas de la Oficina Regional OMS del Pacífico Occidental que relieva el rol de la persona bajo el título "Atención de la Salud: Armonizando la Mente y el Cuerpo, las Personas y los Sistemas" ${ }^{\prime(6)}$.

En el Perú, Honorio Delgado ${ }^{(7)}$ puntualizó en El Medico la Medicina y el Alma lo siguiente: "El positivismo, generalización abusiva de las ideas válidas solo en el dominio de las ciencias físicas, lleva a considerar al enfermo como un objeto material, una cosa y a la medicina como una pura ciencia o una mezcla de ciencia y técnica, por ende, impersonal y mecánica". También en el Perú, Carlos Alberto Seguin ${ }^{(8)}$ ha enfatizado el rol crucial del humanismo en su libro "Tú y la Medicina". Más ampliamente en Latinoamérica, la perspectiva de MCP articulando ciencia y humanismo está tomando creciente cuerpo, tanto conceptualmente ${ }^{(9)}$ como organizacionalmente a través de una Red Latinoamericana de $\mathrm{MCP}^{(10)}$.

\section{Educación Médica Centrada en la Persona}

Appleyard, Ghebrehiwet, Mezzich ${ }^{(11)}$ han puntualizado que, sin una educación universal y transformadora de los profesionales de la salud, es probable que continúe la cultura dominante deshumanizada involucrando enfoques biomédicos reduccionistas y su impacto negativo en la calidad de la atención médica. Por lo tanto, existe la necesidad de optimizar los servicios de salud al reorientar la educación de los profesionales hacia un enfoque centrado en la persona.

Por otro lado, Frenk et al. ${ }^{(12)}$ a nombre de una Comisión Internacional sobre Educación de los Profesionales de la Salud para el Siglo XXI establecida por la revista The Lancet ha planteado "Desarrollar una visión compartida y una estrategia común para la educación postsecundaria en medicina, enfermería y salud pública que va más allá de los límites de las fronteras nacionales y los silos de las profesiones individuales. Este marco integral considera las conexiones entre educación y sistemas de salud. Se centra en las personas como coproductores y como impulsores de necesidades y demandas en ambos sistemas".

\section{Objetivos}

El propósito de este artículo es identificar recomendaciones internacionales contemporáneas para el desarrollo de una educación médica centrada en la persona.

${ }^{1}$ Profesor de Psiquiatría, Icahn School of Medicine at Mount Sinai, New York; Cátedra Hipólito Unanue de Medicina Centrada en la Persona, Universidad Nacional Mayor de San Marcos, Lima; Secretario General, International College of Person Centered Medicine. 


\section{Metodología}

La metodología empleada para la preparación de este artículo ha incluido revisiones selectivas de la literatura y consultas internacionales, particularmente en términos de Declaraciones sobre Educación Médica Centrada en la Persona generadas en los recientes Congresos Internacionales de MCP en Zagreb ${ }^{(13)}$ y en Madrid ${ }^{(14)}$ así como en las secciones sobre Educación Médica Centrada en la Persona dentro del marco de las Jornadas Latinoamericanas de MCP en $\operatorname{Lima}^{(10,15)}$.

\section{Resultados}

El Primer Congreso Internacional de MCP en Zagreb en Noviembre 2013 produjo una Declaración sobre Educación Profesional en Salud Centrada en la Persona. Sus recomendaciones fueron las siguientes:

1. Mejora de las bases conceptuales de la formación profesional y la educación pública al basarlas en la ética y los derechos humanos, el conocimiento de los determinantes biopsico-socioculturales de la salud enferma y la salud positiva, la atención a los valores y preferencias de las personas, y florecimiento de su bienestar y proyectos de vida.

2. Análisis y optimización de la organización y la cultura de las instituciones educativas para ser de apoyo a la salud centrada en la persona para y con los estudiantes, profesores y colegas administrativos.

3. Selección de los estudiantes adeptos a los objetivos y las responsabilidades que se requieren de los profesionales de la salud y el compromiso con su desarrollo personal, además de su amplia formación técnica.

4. Selección de los instructores y tutores comprometidos con sus responsabilidades educativas y su continuo desarrollo profesional y personal.

5. Facilitación de interacciones dinámicas entre los estudiantes, los profesores, los pacientes y las familias, garantizando espacios de diálogo a todos los niveles, promover la formación interdisciplinaria, e incluir a algunos pacientes como instructores.

6. Promover desarrollos curriculares que tengan principios orientadores centrados en la persona, que incluyan experimentos y mecanismos curriculares para su evaluación, que tengan en cuenta el equilibrio entre los bloques concentrados y la integración longitudinal para el logro de los objetivos educativos, y que incorporen una promoción continuada de la salud, la prevención, el tratamiento, la rehabilitación y los cuidados paliativos.

7. Explorar el diseño, implementación y evaluación de los métodos de enseñanza centrados en la persona, como el aprendizaje experiencial y reflexivo, ejercicios de comunicación, la capacitación de grupos con pacientes y familias, el acompañamiento a tutores, utilización de grabaciones de vídeo con fines de retroalimentación, visitas a las comunidades, utilización de las narrativas, la práctica de actividades artísticas, la entrevista motivacional, el establecimiento de una base común entre los médicos, los pacientes y sus familias para el diagnóstico y la atención, la articulación de las competencias y el rendimiento de entrenamiento, y la capacidad para trabajar con pacientes de diferentes culturas y condiciones sociales de una manera respetuosa y colaborativa.

8. Promover la salud en los pacientes individuales y en la sociedad a través de la educación activa, abordando temas generales sobre los determinantes de la salud y el impacto de los factores culturales, religiosos y sociales en la salud y el cuidado de la salud e identificando y reduciendo los riesgos de salud en el medio ambiente y la sociedad, e involucrando a todos en la conformación de políticas de salud acertadas.

El Cuarto Congreso Internacional de MCP en Madrid en Noviembre 2016 generó una Declaración sobre Educación Médica Centrada en la Persona y los Objetivos del Cuidado de la Salud. Sus recomendaciones fueron las siguientes:

1. Las universidades deben asumir la responsabilidad de hacer una selección positiva y justa de sus estudiantes de medicina entre aquellos candidatos que muestren la mejor actitud y cualidades para la práctica centrada en la persona.

2. Es aconsejable incluir asignaciones clínicas tempranamente en estudios de grado, ya que son efectivas para promover la capacidad de empatía de los estudiantes.

3. La educación y la práctica clínicas centradas en la persona deben ir más allá de las limitaciones de la medicina basada en la evidencia, hacia una medicina holística basada en la evidencia y enriquecida por la consideración de la experiencia y los valores para la toma de decisiones clínicas.

4. Los graduados de medicina deben poder resolver problemas de creciente complejidad a través del razonamiento clínico básico y habilidades prudentes de toma de decisiones en situaciones inciertas, en las cuales la consideración de las expectativas y preferencias del paciente y su familia es esencial.

5. Los servicios de atención primaria de salud representan un entorno ideal para esta experiencia clínica temprana debido a la naturaleza inclusiva y continua de la atención y al establecimiento de relaciones clínicas prolongadas entre los pacientes y sus cuidadores de salud.

6. Los centros educativos deben tener disponible la infraestructura y el personal experto en diferentes simulaciones técnicas (robótica, realidad virtual, cirugía experimental, etc.) que brinden apoyo técnico a educadores clínicos.

7. Todas las universidades y los proveedores de educación deben tener acceso a unidades de educación médica especializadas, que brinden apoyo a los programas, capaciten al personal docente clínico y evalúen la calidad del trabajo realizado. 
8. La educación médica centrada en la persona debe incluir en sus contenidos algunos criterios generales para evaluar los resultados de las intervenciones médicas, como la efectividad clínica, la seguridad y los costos, y la perspectiva del paciente, incluidas las medidas de resultados informados por los pacientes (PROM) y las medidas de experiencia informadas por los pacientes (PREM).

9. La acreditación y la recertificación de los médicos en ejercicio deben basarse en actividades de aprendizaje permanente de gran calidad y amplio alcance.

10. Se deben investigar las metodologías más apropiadas en las disciplinas humanísticas (antropología, ética, derechos humanos, literatura, historia, deontología y legislación sanitaria, pedagogía, artes, etc.) para que dichas áreas temáticas sean significativas, aceptables y beneficiosamente enseñadas a los estudiantes.

La Primera Jornada Latinoamericana de MCP en Lima en Diciembre 2015 produjo una Declaración de Lima sobre Desarrollo Latinoamericano para una Medicina Centrada en la Persona: Del Concepto a la Acción. Entre sus recomendaciones están las siguientes focalizadas sobre Educación Médica Centrada en la Persona:

1. Reconocer y tratar estratégicamente los factores que han contribuido sistemáticamente a la deshumanización de la medicina y al despilfarro de sus avances científicos. Entre estos problemas y oportunidades perdidas se encuentran el debilitamiento de la relación médico-paciente, el tiempo inadecuado y la burocratización de los encuentros clínicos, la proliferación imprudente de las escuelas de medicina y la comercialización y la superficialización de la atención médica.

2. Avanzar en el estudio de las bases y perspectivas latinoamericanas hacia la atención centrada en la persona, basada en imperativos éticos y enfoques científicos sólidos e integrales. De acuerdo con el principio kantiano de que una persona es siempre un fin y no un medio, la MCP surge como una forma de profundizar el estudio y la organización de acciones de salud en la atención clínica, educación médica, investigación científica y salud pública.

3. Avanzar en el estudio, la aplicación y la evaluación de los principios de MCP, incluidos el compromiso ético, el marco holístico, la conciencia cultural y la capacidad de respuesta, la comunicación y el enfoque relacional, los programas de atención clínica de salud individualizados, el establecimiento de una base común entre los clínicos, los pacientes y las familias para el diagnóstico conjunto. comprensión y toma de decisiones compartidas, organización de servicios integrados centrados en personas y comunidades, e investigación médica e investigación científica sobre salud centrada en la persona en su totalidad.

4. Promover el desarrollo de una metodología de educación médica y de salud centrada en la persona que tenga en cuenta los principios de MCP. Esto puede incluir el diseño y la evaluación de experimentos curriculares cuidadosamente pensados, y la selección y desarrollo de estudiantes y profesores que prestan atención a los objetivos personales, éticos y profesionales, junto con la exploración de programas de tutoría de alta calidad.

5. Cultivar la metodología de la investigación científica para la medicina centrada en la persona y la salud. Un principio fundamental es reconocer que cualquier investigación, para ser ética, debe estar centrada en la persona. Esto requiere no solo la aplicación de códigos como la Declaración de Helsinki de la Asociación Médica Mundial, sino también mecanismos que refuercen su implementación como recomienda la Organización Mundial de la Salud. También es necesario que la investigación médica atienda las experiencias, valores y expectativas de los sujetos involucrados y atienda las necesidades de salud de la sociedad en general.

6. Desarrollar programas y políticas de salud pública centrado en la persona y en las personas. La experiencia y creatividad latinoamericana puede enriquecer el camino a seguir. Las metas a considerar abarcan las estrategias actuales de la Organización Mundial de la Salud hacia servicios centrados en las personas intra-e inter-sectoriales que priorizan la atención primaria y la cobertura universal de salud.

7. Promover el cultivo de MCP, sus principios y prácticas según se apliquen de manera coordinada a la atención clínica, la capacitación médica, la educación para la salud, la investigación científica y la salud pública.

8. Organizar a través de la Red Latinoamericana de Medicina Centrada en la Persona, en colaboración con las instituciones nacionales e internacionales disponibles, encuentros y reuniones periódicas para promover intercambios de experiencias y perspectivas estimulantes. Estas actividades se optimizarían mediante la comunicación bilateral y fluida con organizaciones internacionales como la Asociación Latinoamericana de Academias Nacionales de Medicina (ALANAM), la Organización Panamericana de la Salud (OPS / OMS), la Asociación Médica Mundial (AMM), y el Colegio Internacional de Medicina Centrada en la Persona (ICPCM).

La Segunda Jornada Latinoamericana de MCP en Lima en Diciembre 2015 produjo una Declaración de Lima sobre Ciencia y Humanismo en Medicina Centrada en la Persona en Latinoamérica. Entre sus recomendaciones están las siguientes focalizadas sobre Educación Médica Centrada en la Persona:

1. La educación médica debiera incluir el fortalecimiento de los contenidos humanísticos del currículo y el temprano, frecuente y profundo contacto del estudiante con personas y familias. Mejoras curriculares deben abarcar integraciones interdisciplinarias y énfasis en nuevas y cruciales áreas como la atención primaria a la salud.

2. Dada la necesidad impostergable del trabajo en equipo para una atención clínica cabal, debe incluirse en la formación de los profesionales de la salud oportunidades amplias para el aprendizaje inter-profesional. Esto debe involucrar a las más importantes disciplinas como medicina, enfermería, obstetricia, psicología, salud pública, nutrición, rehabilitación, educación y trabajo social. 
3. Es crítico el uso de promisores métodos educacionales. Entre ellos está el modelaje vivo ejercido por el profesor, que es crucial e insustituible. En palabras de Albert Einstein, "Dar el ejemplo no es la principal manera de influir en los demás; es la única". Otro es la diversificación de los ambientes educacionales, para adiestrar al estudiante en las diversas realidades clínicas y comunitarias donde deberá ejercer profesionalmente.

4. El compromiso ético es la primera característica denotativa de la medicina centrada en la persona. En línea con ello, debe incrementarse la formación ética del estudiante a través de un eje de oportunidades educacionales a lo largo de la carrera. Deben incorporarse cursos pertinentes, privilegiando la discusión y reflexión sobre casos ilustrativos, y el irremplazable ejemplo de moralidad y respeto por la persona del paciente y del estudiante que deben ofrecer los profesores.

5. Otra área formativa fundamental es adiestramiento en comunicación clínica, dado el profundo valor humano del encuentro entre médico y paciente, y las oportunidades ofrecidas por la creciente experiencia latinoamericana con metodología pertinente. Particularmente clave aquí es la enseñanza sobre cómo establecer empatía.

6. Se deben crear programas de tutoría integral, a través de los cuales se promueva el desarrollo del estudiante como persona y profesional. Los tutores participantes deben estar debidamente entrenados y su desarrollo como profesionales de la salud, educadores universitarios y personas cabales debe ser promovido activamente por su institución académica

\section{Análisis de Patrones Conceptuales}

Un análisis de patrones conceptuales en los grupos de recomendaciones listadas arriba para el desarrollo de una educación médica centrada en la persona provenientes de cuatro eventos mundiales o latinoamericanos permite formular las siguientes como recomendaciones principales, las cuales han sido elaboradas adicionalmente en artículos académicos acompañantes a las Declaraciones ${ }^{(9,11,16,17)}$ :

1. Basamento en los principios y estrategias de la medicina centrada en la persona (MCP) tales como compromiso ético, marco holístico, conciencia y responsabilidad cultural, matriz relacional, atención individualizada, base común entre clínicos, paciente y familia para diagnóstico y acción compartidos, servicios de salud integrados y centrados en la comunidad, y educación e investigación de salud centrados en la persona.

2. Establecimiento de una cultura organizacional centrada en la persona, ajena al comercialismo que en tiempos recientes ha llevado en algunos países a la proliferación de facultades de medicina de cuestionable calidad.

3. Selección de estudiantes y profesores con características personales y profesionales cabales.

4. Desarrollo de programas de tutoría enderezados a la formación personal y profesional de los estudiantes, con la participación de profesores profesionalmente y pedagógicamente competentes y dignos modelos de calidad humana.

5. Diseño, implementación y evaluación curricular centrados en la persona. Tales currículos deben incluir cursos de humanidades y ciencias sociales, en adición a los biomédicos, adiestramiento en capacidades para tomar decisiones éticas, juiciosas y prudentes, y actitudes respetuosas de la dignidad y autonomía de los pacientes, y empoderativas de familias y comunidades. Tales currículos deben incluir temprano contacto con pacientes y comunidad y ser sujetos de experimentos adecuadamente evaluados. Currículos centrados en la persona son necesarios para el pre-grado, post-grado (residencias) y educación médica continua.

6. Desarrollo de programas de formación interprofesional, que asegure la atención a la salud centrada en la persona por equipos que incluyan médicos, enfermeras, psicólogos, obstetrices, farmacéuticos, nutricionistas y fisioterapeutas entre otros profesionales de la salud.

7. Diseño, implementación y evaluación de actividades educativas tales como ejercicios comunicacionales, entrevistas motivacionales, sesiones colaborativas para el diagnóstico y las decisiones terapéuticas, simulaciones educativas, aprendizaje reflexivo, trabajo en grupo con pacientes y familiares, sesiones de acompañamiento al profesor, videograbaciones, y actividades narrativas y artísticas.

8. Empleo de ambientes de aprendizaje diversos que reflejen el amplio campo de actividades de los profesionales de la salud, considerando prominentemente a la atención primaria y ambulatoria.

9. Desarrollo de infraestructura institucional adecuada para una educación médica centrada en la persona, tales como instalaciones y personal apropiados para simulaciones en comunicación, diagnóstico y tratamiento y unidades educativas para asesorar y evaluar la formación profesional.

10. Programas de investigación para el desarrollo de nueva metodología educativa y su evaluación sistemática.

\section{Discusión}

Una revisión selectiva de la literatura reciente sobre educación médica contemporánea ofrece interesantes comentarios a las recomendaciones sobre educación médica centrada en la persona presentadas en la sección de resultados.

La autoritativa Federación Mundial para la Educación Médica plantea por un lado la incorporación en los currículos de las Escuelas de Medicina una serie de asignaturas no siempre consideradas tales como ética médica, psicología médica, salud mental, sociología médica entre otras tal como se consigna en las recomendaciones listadas. En relación adicional con el currículo previene contra la especialización demasiado temprana. También concurre en recomendar el entrenamiento en destrezas básicas tales como la comunicación clínica $^{(18)}$. 
Un grupo internacional de jóvenes profesionales de la salud $^{(19)}$ proponen que el desarrollo profesional óptimo en medicina se relacione fundamentalmente con los principios y estrategias prácticas de medicina centrada en la persona. Postulan también un proceso bidireccional y reflexivo involucrando a profesores y estudiantes que guarda relación con las recomendaciones brevemente listadas sobre tutoría y desarrollo profesional de estudiantes y profesores.

Un proyecto búlgaro de salud pública ${ }^{(20)}$ considera que la política global de la educación contemporánea en medicina está dominada por un marco y contenido cognitivo tecnocrático. En respuesta, proponen la introducción del modelo de atención centrada en la persona en la política global de educación médica en armonía con avances internacionales en este campo.

Manuel Dayrit, ex ministro de Salud de las Filipinas y ex Director de Educación Profesional en la OMS arguye que la ampliación y transformación de la educación profesional de la salud resuena con los esfuerzos actuales para centrar la atención médica en la persona y la salud pública en las personas. Tal compromiso continuo entre todas las partes interesadas en este empeño representa una ampliación transformadora de la educación profesional en salud ${ }^{(21)}$.

Una comisión internacional liderada por Frenk et al. ${ }^{(12)}$ propone lineamientos para la formación de profesionales de la salud pertinentes para el presente siglo. Entre estos lineamientos están los siguientes: las reformas educativas deben adoptar enfoques basados en competencias para el diseño instruccional local teniendo en cuenta recursos globales; promover la educación interprofesional y transprofesional que rompa los silos profesionales y busque colaboración no jerárquica en equipos efectivos; aprovechar el poder de la tecnología de la información para el aprendizaje; fortalecer los recursos educativos, con especial énfasis en el desarrollo del profesorado; y promover un nuevo profesionalismo que desarrolle un conjunto común de valores en torno a la responsabilidad social.

\section{Conclusiones}

Mucho se ha avanzado en tiempos recientes en principios y métodos para una educación médica centrada en la persona, pero queda mucho por hacer a través de procesos colaborativos locales e internacionales que articulen ciencia y humanismo.

Pasar del modelo biomédico actual centrado en los órganos y las enfermedades hacia un paradigma bio-psicosociocultural más amplio e inclusivo, implica una forma más profunda de pensar sobre la medicina y la salud focalizándolas en la persona individual y las personas en la comunidad. Esto podrá llevar a un despertar en las instituciones educativas para apoyar la formación cabal de los profesionales de la salud y alentará a los estudiantes impulsados por su vocación a unirse en un entorno de aprendizaje que fomente una medicina y salud centradas en las personas ${ }^{(11)}$.

Referencias bibliográficas

1.Mezzich JE. Psychiatry for the Person: Articulating Medicine's Science and Humanism. World Psychiatry. 2007;6:65-67.

2.WHO. World Health Report. Geneva, 2008 Author.

3. World Health Organization. World Health Assembly Resolutions on Primary Health Care, Including Health System Strengthening, WHA 62.12, 22 May 2009.

4.Mezzich JE, Snaedal J, van Weel C, Heath I. Conceptual Explorations on Person-centered Medicine. International Journal of Integrated Care. Vol 10. Supplement, 2010.

5. Mezzich JE, Snaedal J, van Weel C, Heath I. Toward Person-centered Medicine: From disease to patient to person. Mount Sinai Journal of Medicine 2010;77:304-306.

6.WHO Western Pacific Regional Office. Health Care Harmonizing Mind and Body, Persons and Systems. Author, Geneva, 2007.

7.Delgado H. El Médico, la Medicina y el Alma. Universidad Peruana Cayetano Heredia, Lima, 1992.

8. Seguín CA. Tu y la Medicina. Editorial Poniente, Lima, 1993.

9. Wagner P, Perales A, Arms R, Codas O, Saints R, Elio-Calvo D, Mendoza-Vega J, Arce M, Calderón JL, Llosa L, Saavedra J, Ugarte O, Vildózola H, Mezzich JE. An Fac med 2015;76:63-70.

10. Latin American Network for Person Centered Medicine. Lima Declaration 2016. Science and Humanism in Person Centered Medicine in Latin America. International Journal of Person Centered Medicine 2017;7 (3):147-150.

11. Appleyard J, Ghebrehiwet T, Mezzich JE. Development and implications of the Zagreb Declaration on Person-centered Health Professional Education. International Journal of Person Centered Medicine 2014;4(1):8-13.

12. Frenk J, Chen L, Butta $\mathbf{Z}$, et al. Health professionals for a new century: transforming education to strengthen health systems in an interdependent world. The Lancet. 2010;376(9756):1923-1958.
13. International College of Person Centered Medicine. Zagreb Declaration on Person Centered Health Professional Education. International Journal of Person Centered Medicine 2014;4(1):6-7.

14. International College of Person Centered Medicine. Madrid Declaration on Person Centered Medical Education and the Goals of Healthcare. International Journal of Person Centered Medicine 2017;7(2):80-81.

15. Latin American Network for Person Centered Medicine. The 2015 Lima Declaration on Latin American Developments for Person Centered Medicine. International Journal of Person Centered Medicine 2016;6(1):7-8

16. Appleyard A, Botbol N, Caballero F, Ghebrehiwet T, Mezzich JE, Perez-Miranda $\mathbf{J}$ et al. Bases of the Madrid Declaration on Person Centered Medical Education to Enhance Health Systems Worldwide. International Journal of Person Centered Medicine 2017;7(2):82-90.

17. Cluzet O, Perales A, Canchihuamán F, Mezzich JE. Latinamerican Experiences and Reflections on Science and Humanism in Person Centered Medicine. International Journal of Person Centered Medicine 2017;7(3):151-159.

18. Karle H. World Federation for Medical Education. International Journal of Integrated Care $2010 ; 10(5)$ : DOI: http://doi.org/10.5334/ijic.477.

19. Christodoulou N, Ramalho R, Larsson M, Karpenko O. Personcentered Medicine and Professional Development. International Journal of Person Centered Medicine. 2011;1(3):581-584.

20.Stoyanov D, Hristov J, Tornyova B, Dimitrova D. Bulgarian Person centered Public Health Project: Towards introduction of the person centered care model in the global medical education policy Int J Person Centered Med 2011,1:101-104.

21.Dayrit M. Transformative scale-up of health professional education International Journal of Person Centered Medicine 2012;2(2):161-164. 